\title{
ON THE ERGODIC THEOREM IN THE KOZLOV-TRESHCHEV FORM
}

\author{
V.I. BOGACHEV, A.V. KOROLEV
}

\begin{abstract}
Given an ergodic semigroup of transformations $T_{t}$ of a probability space $(X, \mathcal{A}, \mu)$, we introduce and study measures on $X$ induced by mappings $s \mapsto T_{t s} x$ from $[0,+\infty)$ to $X$ and probability measures on $[0,+\infty)$. We obtain several results related the ergodic theorem in the form found recently by Kozlov and Treshchev.
\end{abstract}

AMS Subject Classification: 28D10, 37A30

Let $(X, \mathcal{A}, \mu)$ be a probability space, let $\mathcal{A}_{\mu}$ be the completion of $\mathcal{A}$ with respect to $\mu$, and let $\left\{T_{t}\right\}_{t \geq 0}$ be a semigroup of transformations of the space $X$ (i.e., $T_{0}=I$, $\left.T_{t} T_{s}=T_{t+s}\right)$ preserving the measure $\mu$, and let the mapping

$$
(t, x) \mapsto T_{t}(x), \quad[0,+\infty) \times X \rightarrow X,
$$

be measurable with respect to the pair of $\sigma$-algebras $\mathcal{B}([0,+\infty)) \otimes \mathcal{A}_{\mu}$ and $\mathcal{A}$, where $\mathcal{B}([0,+\infty))$ is the Borel $\sigma$-algebra of $[0,+\infty)$. The semigroup $\left\{T_{t}\right\}$ is also called a semiflow. The invariance of the measure $\mu$ with respect to $T_{t}$ means that the image of the measure $\mu$ under the mapping $T_{t}$ coincides with $\mu$, i.e., for every bounded $\mathcal{A}$-measurable function $\varphi$, one has the equality

$$
\int_{X} \varphi\left(T_{t}(x)\right) \mu(d x)=\int_{X} \varphi(x) \mu(d x), \quad t \geq 0 .
$$

Let $f$ be a bounded $\mathcal{A}$-measurable function. In papers [1] and [2], V.V. Kozlov and D.V. Treshchev have shown that, for every absolutely continuous probability measure $\nu$ on $[0,+\infty)$ with a density $\varrho$ with respect to Lebesgue measure, the functions

$$
F_{t}(x):=\int_{0}^{\infty} f\left(T_{t s}(x)\right) \nu(d s)
$$

converge to a limit $\bar{f}(x)$ for $\mu$-almost all $x$ as $t \rightarrow+\infty$, where $\bar{f}$ is the function to which, by the Birkhoff-Khinchin ergodic theorem (see [3], Ch. 1, 2 ), the time averages

$$
\frac{1}{t} \int_{0}^{t} f\left(T_{s}(x)\right) d s
$$

converge for $\mu$-almost all $x$ as $t \rightarrow+\infty$. We recall that $\bar{f}$ is the conditional expectation of $f$ with respect to the $\sigma$-algebra $\mathcal{T}$ generated by all $\mu$-measurable functions $\varphi$ with the following property: for every $\tau \geq 0$, one has $\varphi\left(T_{\tau}(x)\right)=\varphi(x)$ for $\mu$-almost all $x \in X$. If the dynamic system under consideration is ergodic, then $\bar{f}$ is the constant equal to the integral of $f$ with respect to the measure $\mu$, i.e.,

$$
\lim _{t \rightarrow+\infty} \int_{0}^{+\infty} f\left(T_{t s}(x)\right) \nu(d s)=\int_{X} f(y) \mu(d y) .
$$


Although this interesting result is readily deduced from the individual ergodic theorem (which is equivalent to the case where $\nu$ is Lebesgue measure on the interval $[0,1])$, it is very unexpected, since, at the first glance, the character of averaging in it completely differs from the classic uniform averaging. The observation of V.V. Kozlov and D.V. Treshchev motivates a consideration of the family of measures $\nu_{t, x}$ on $X$ defined as follows: the measure $\nu_{t, x}$ is the image of the measure $\nu$ under the mapping

$$
S_{t, x}:[0,+\infty) \rightarrow X, \quad S_{t, x}(s):=T_{t s}(x),
$$

i.e., the integral of any $\mathcal{A}$-measurable bounded function $\varphi$ against the measure $\nu_{t, x}$ is given by the formula

$$
\int_{X} \varphi(z) \nu_{t, x}(d z)=\int_{0}^{\infty} \varphi\left(T_{t s}(x)\right) \nu(d s) .
$$

The aim of our work is a study of the character of convergence of the measures $\nu_{t, x}$ to the measure $\mu$. In addition, we discuss the case of not necessarily absolutely continuous measures $\nu$. Finally, we obtain a relationship between the orders of the integrability of the functions $f$ and $\varrho$ that ensures the validity of the conclusion of the Kozlov-Treshchev theorem. In particular, it turns out that the boundedness of $f$ cannot be removed without additional assumptions even if the density $\varrho$ has bounded support; however, a simultaneous boundedness of the density $\varrho$ and of its support enables one to take any $\mu$-integrable functions $f$.

First we consider several examples related to singular measures $\nu$ and unbounded functions $f$. It should be noted at once that the existence of atoms of the measure $\nu$ excludes convergence of $F_{t}(x)$ in many cases. For example, if $\nu$ is Dirac's measure at the point 1 , then $F_{t}(x)=f\left(T_{t}(x)\right)$. If $T_{t}$ is the rotation of the circle in an angle $t$, then there is no limit $T_{t}(x)$ as $t \rightarrow \infty$, hence it is easy to find a continuous function $f$ for which at no $x$ has $F_{t}(x)$ a limit. The case of an atomless measure is more interesting.

Example 1. Let $X$ be the unit circle with its normalized Lebesgue measure $\mu$ and let $T_{t}$ be the rotation of the circle in an angle $-t$. There exists an atomless singular Borel probability measure $\nu$ on $[0,1]$ such that for the function $f(z)=z$, where $z=\exp (i \theta), \theta \in[0,2 \pi)$, for no $z \in X$ has the quantity $F_{t}(z)$ a limit as $t \rightarrow \infty$.

Proof. It is known (see [4], §2.2) that there exists an atomless singular Borel probability measure $\nu$ on $[0,1]$ such that its Fourier transform $\widehat{\nu}$ has no limit at infinity. It remains to observe that $F_{t}(z)=\sqrt{2 \pi} z \widehat{\nu}(t)$.

The same system shows that a finite limit of $F_{t}(x)$ may not exist for an unbounded function $f$ and an absolutely continuous measure $\nu$.

Example 2. Let $\mu$ and $T_{t}$ be the same as in the previous example. There exist a $\mu$ integrable Borel function $f$ on $X$ and an absolutely continuous probability measure $\nu$ with support in the interval $[0,1]$ such that we have $\lim \sup _{n \rightarrow \infty} F_{n}(z)=+\infty$ for all $z$.

Proof. Let us define a probability measure $\nu$ by a density $\varrho$ concentrated on the set $\bigcup_{k=4}^{\infty}\left[k^{-1}-k^{-3}, k^{-1}\right]$ by letting

$$
\varrho(s):=c k^{2}(\ln k)^{-2} \quad \text { if } s \in\left[k^{-1}-k^{-3}, k^{-1}\right], k \geq 4 .
$$

At all other points in $[0,1]$ we set $\varrho(s)=0$; the constant $c$ is chosen in such a way that $\nu$ be a probability measure. Let

$$
f(z)=|\sin \theta|^{-1}(\ln |\sin \theta|-1)^{-2} \quad \text { if } z=\exp (i \theta), \theta \in[0,2 \pi) .
$$


Then

$$
\begin{aligned}
F_{n}(z) & =\int_{0}^{1} f(\exp (\text { ins }-i \theta)) \varrho(s) d s \\
& =\sum_{k=4}^{\infty} c k^{2}(\ln k)^{-2} \int_{1 / k-1 / k^{3}}^{1 / k}|\sin (n s-\theta)|^{-1}(\ln |\sin (n s-\theta)|-1)^{-2} d s \\
& =\sum_{k=4}^{\infty} c k^{2} n^{-1}(\ln k)^{-2} \int_{n / k-n / k^{3}}^{n / k}|\sin (u-\theta)|^{-1}(\ln |\sin (u-\theta)|-1)^{-2} d u .
\end{aligned}
$$

It is known (see $[5], \S 8$ ) that for every $\theta \in[0,2 \pi$ ), there exist infinitely many pairs of natural numbers $n$ and $k$ for which $|\theta-n / k| \leq k^{-2}$. For such $\theta, n, k$ we obtain that $n / k \leq 2 \pi+1$ and $|u-\theta| \leq(2 \pi+2) k^{-2} \leq 9 k^{-2}$ for all $u \in\left[n / k-n / k^{3}, n / k\right]$. Therefore, we have

$$
\begin{aligned}
F_{n}(z) & \geq c k^{2} n^{-1}(\ln k)^{-2} \int_{n / k-n / k^{3}}^{n / k}|\sin (u-\theta)|^{-1}(\ln |\sin (u-\theta)|-1)^{-2} d u \\
& \geq c k^{2} n^{-1}(\ln k)^{-2} \int_{n / k-n / k^{3}}^{n / k} 9^{-1} k^{2}\left|\ln \left(9 / k^{2}\right)-1\right|^{-2} d u \\
& =\frac{c}{9} k(\ln k)^{-2}|\ln 9-2 \ln k-1|^{-2} .
\end{aligned}
$$

Hence $\lim \sup _{n \rightarrow \infty} F_{n}(z)=+\infty$. We can also take $f(z)=|\sin \theta|^{-p}, p \in(1 / 2,1)$, or even $f(z)=|\sin \theta|^{-1 / 2}(\ln |\sin \theta|)^{3}$ (however, see Theorem 3).

Let us note that in the case of an ergodic system, the result of V.V. Kozlov and D.V. Treshchev has some similarity with convergence of the measures $\nu_{t, x}$ to the measure $\mu$ in the topology of convergence on every set. However, there might be no such convergence in its literal meaning, since we obtain convergence for almost all $x$ after integrating a fixed function $f$; so the corresponding measure zero set may depend on $f$. For example, let us consider the ergodic group $\left\{T_{t}\right\}$ of shifts along the trajectories of the differential equation $d z_{1} / d t=\alpha_{1}, d z_{2} / d t=\alpha_{2}$ (with incommensurable $\alpha_{1}$ and $\alpha_{2}$ ) on the two-dimensional torus $S^{2}$ with its normalized Lebesgue measure $\mu$, which is invariant with respect to $T_{t}$. For every Borel probability measure $\nu$ on $[0,+\infty)$, all measures $\nu_{t, x}$ are singular with respect to the measure $\mu$, since every measure $\nu_{t, x}$ is concentrated on a curve of $\mu$-measure zero. Hence, for no $x$, converge these measures to $\mu$ on every set (although, for absolutely continuous measures $\nu$, weak convergence holds, as we shall see below). It is interesting to find sufficient conditions for convergence of the measures $\nu_{t, x}$ to $\mu$ in the topology of convergence on every set or in the variation norm. Basic facts related to Radon measures on topological spaces and weak convergence of measures that we use below can be found in book [6], v. 2 .

Theorem 1. Let $\mu$ be a Radon probability measure on a completely regular topological space $X$ such that all compact sets in $X$ are metrizable. Suppose that the semiflow $\left\{T_{t}\right\}$ is ergodic. Let the measure $\nu$ be absolutely continuous. Then, for $\mu$-almost all $x$, the measures $\nu_{t, x}$ converge weakly to $\mu$ as $t \rightarrow \infty$.

Proof. Let $f$ be a bounded Borel function on $X$. By the ergodicity, for $\mu$-almost all $x$ we have

$$
\lim _{t \rightarrow \infty} \int_{X} f(z) \nu_{t, x}(d z)=\int_{X} f(z) \mu(d z)
$$

For every natural number $n$ we choose a compact set $K_{n}$ such that $\mu\left(X \backslash K_{n}\right)<2^{-n}$. Letting $f=I_{K_{n}}$ we obtain that there exists a set $X_{1} \subset X$ with the following 
property: $\mu\left(X_{1}\right)=1$ and for every $x \in X_{1}$ there exists $t_{n}(x)$ such that, for all $t>t_{n}(x)$, the inequality $\nu_{t, x}\left(X \backslash K_{n}\right)<2^{-n}$ holds. Let us choose a countable family $\left\{f_{n, k}\right\}$ of continuous functions on $K_{n}$ that is everywhere dense in the unit ball of the space $C\left(K_{n}\right)$. Since $X$ is completely regular, we can extend $f_{n, k}$ to a continuous function on $X$, denoted by the same symbol, that satisfies the condition $\left|f_{n, k}(x)\right| \leq 1$. There exists a set $X_{0} \subset X_{1}$ such that $\mu\left(X_{0}\right)=1$ and, for every $x \in X_{0}$ and all $n$ and $k$, the integrals of the functions $f_{n, k}$ with respect to the measure $\nu_{t, x}$ converge to the integral of the function $f_{n, k}$ with respect to the measure $\mu$ as $t \rightarrow+\infty$. Let us show that, for all $x \in X_{0}$, the measures $\nu_{t, x}$ converge weakly to $\mu$ as $t \rightarrow+\infty$. Let $f$ be a bounded continuous function on $X$ with $|f(x)| \leq 1$. Let $\varepsilon \in(0,1)$. Let us take $n$ with $2^{-n} \leq \varepsilon$. One can find a number $k$ such that $\left|f(x)-f_{n, k}(x)\right| \leq \varepsilon$ if $x \in K_{n}$. Then, whenever $x \in X_{0}$ and $t>t_{n}(x)$, we have

$$
\int_{X}\left|f(z)-f_{n, k}(z)\right| \nu_{t, x}(d z) \leq 2 \varepsilon, \quad \int_{X}\left|f(z)-f_{n, k}(z)\right| \mu(d z) \leq 2 \varepsilon .
$$

This yields the estimate

$$
\limsup _{t \rightarrow+\infty}\left|\int_{X} f(z) \nu_{t, x}(d z)-\int_{X} f(z) \mu(d z)\right| \leq 4 \varepsilon,
$$

which proves our assertion because $\varepsilon$ is arbitrary.

The statement of this theorem may be true for some singular measures $\nu$ as well. However, we do not know whether there exists a continuous singular measure $\nu$ for which the Kozlov-Treshchev theorem remains valid for all bounded measurable functions $f$ (not necessarily continuous).

Example 3. In the situation of Example 1, there exists an atomless singular Borel probability measure $\nu$ on $[0,1]$ such that, for every $x$, the measures $\nu_{t, x}$ converge weakly to $\mu$ as $t \rightarrow \infty$.

Proof. It is known that there exists an atomless singular Borel probability measure $\nu$ on $[0,1]$ such that its Fourier transform $\widehat{\nu}$ tends to zero at infinity (see [4], §2.2). This shows that for every function $f$ of the form $f(z)=\alpha z^{k}+\beta \bar{z}^{m}$ with $k, m \in \mathbb{N}$, the equality

$$
\lim _{t \rightarrow \infty} \int_{X} f(z) \nu_{t, x}(d z)=0
$$

holds for every $x \in X$. Therefore, for every polynomial $f$ in $z$ and $\bar{z}$, on account of the equality $z \bar{z}=1$ on $X$, we obtain equality (1). This proves weak convergence of $\nu_{t, x}$ to $\mu$.

Remark 1. Let $\mu$ be a Radon measure on a completely regular space $X$ and let $\nu$ be a Borel probability measure on the real line (possibly singular) such that, for every bounded continuous function $f$ on $X$, equality (1) holds for $\mu$-almost all $x$. Then, for every Borel function $g \in L^{1}(\mu)$, one has the equality

$$
\lim _{t \rightarrow+\infty} \int_{X}\left|\int_{0}^{\infty} g\left(T_{t s}(x)\right) \nu(d s)-\int_{X} g(z) \mu(d z)\right| \mu(d x)=0 .
$$

Indeed, let us fix $\varepsilon>0$. Let us find a bounded continuous function $f$ on $X$ such that $\|f-g\|_{L^{1}(\mu)} \leq \varepsilon$. It follows from our assumption and Lebesgue's dominated convergence theorem that there exists $\tau>0$ such that for all $t \geq \tau$ one has the inequality

$$
\int_{X}\left|\int_{0}^{\infty} f\left(T_{t s}(x)\right) \nu(d s)-\int_{X} f(z) \mu(d z)\right| \mu(d x) \leq \varepsilon .
$$


It remains to use the estimate

$$
\int_{X} \int_{0}^{\infty}\left|f\left(T_{t s}(x)\right)-g\left(T_{t s}(x)\right)\right| \nu(d s) \mu(d x) \leq \varepsilon,
$$

which follows from Fubini's theorem and the invariance of $\mu$. In the situation under consideration, one can choose a sequence $t_{n}$ increasing to infinity such that, as $n \rightarrow+\infty$, the sequence $F_{t_{n}}(x)$ will converge $\mu$-almost everywhere to the integral of the function $f$ against the measure $\mu$.

Remark 2. Suppose that $X$ is a locally compact space in which all compact sets are metrizable. Let $\mu$ be a Radon probability measure and let the semiflow $\left\{T_{t}\right\}$ be ergodic. Let $\nu$ be a Borel probability measure on the real line (possibly singular) such that, for every bounded continuous function $f$ on $X$, equality (1) holds for $\mu$-almost all $x$. Then, for $\mu$-almost all $x$, the measures $\nu_{t, x}$ converge weakly to the measure $\mu$ as $t \rightarrow+\infty$. This is clear from the proof of Theorem 1 taking into account that here, too, there exist compact sets $K_{n}$ with the property that, for $\mu$ almost every $x$, there exists $t_{n}(x)$ such that for all $t>t_{n}(x)$ one has the inequality $\nu_{t, x}\left(X \backslash K_{n}\right)<2^{-n}$. Indeed, by the local compactness of $X$, there exist continuous functions $\psi_{n}: X \rightarrow[0,1]$ with compact supports $K_{n}$ such that the integral of $\psi_{n}$ with respect to the measure $\mu$ tends to 1 .

We recall that a family $\mathcal{M}$ of nonnegative Borel measures on a topological space is called uniformly tight if, for every $r>0$, there exists a compact set $K_{r} \subset X$ such that $m\left(X \backslash K_{r}\right)<r$ for all $m \in \mathcal{M}$.

Theorem 2. Suppose that $X$ is a Souslin (or metric) space and $\mu$ is a Radon probability measure. Let the semiflow $\left\{T_{t}\right\}$ be ergodic. Let the measure $\nu$ be absolutely continuous. Then, for every $\varepsilon>0$, there exists a compact set $X_{\varepsilon} \subset X$ such that $\mu\left(X_{\varepsilon}\right)>1-\varepsilon$ and the family of measures $\nu_{t, x}$ with $t \geq \varepsilon$ and $x \in X_{\varepsilon}$ is uniformly tight.

Proof. Since the measure $\nu$ can be approximated in the variation norm by a sequence of measures with bounded support and bounded densities, it suffices to prove our claim for the normalized Lebesgue measure on the interval $[0, a]$. For notational simplicity we shall assume that $a=1$. Let $\varepsilon<1$. Let us find compact sets $K_{n}$ such that $\mu\left(K_{n}\right)>1-\varepsilon^{2} 4^{-n}$. It follows by the ergodicity of $\left\{T_{t}\right\}$ that, for $\mu$-almost all $x$, we have

$$
\lim _{m \rightarrow+\infty} \sup _{t \geq m}\left|\nu_{t, x}\left(K_{n}\right)-\mu\left(K_{n}\right)\right|=0 .
$$

By Egoroff's theorem, there exist compact sets $C_{n} \subset X$ and numbers $m_{n}$ such that

$$
\mu\left(C_{n}\right)>1-\varepsilon^{2} 4^{-n}, \quad \nu_{t, x}\left(K_{n}\right)>1-\varepsilon^{2} 4^{-n} \quad \text { if } x \in C_{n}, t \geq m_{n} .
$$

Let us fix $n$ and set $\tau=m_{n}$. Let $t \in[0, \tau], x \in X$. Let $\lambda$ denote Lebesgue measure on $[0, \tau]$. Let us consider the mapping $F:(t, x) \mapsto T_{t}(x)$. By Lusin's theorem (which is applicable because every Borel measure on the Souslin space $X$, in particular, the image of the measure $\lambda \otimes \mu$ under the mapping $F$, is concentrated on a countable union of metrizable compact sets), there exists compact sets $Q_{n} \subset[0, \tau] \times X$ such that the mapping $F$ is continuous on $Q_{n}$ and

$$
\lambda \otimes \mu\left(Q_{n}\right)>\tau-\varepsilon^{2} 4^{-n} .
$$

The set $F\left(Q_{n}\right)$ is compact. Let $Q_{n}^{x}:=\left\{t:(t, x) \in Q_{n}\right\}$. There exist compact sets $E_{n} \subset X$ with $\mu\left(E_{n}\right)>1-\varepsilon 2^{-n}$ such that, for every $x \in E_{n}$, the inequality 
$\lambda\left(Q_{n}^{x}\right)>\tau-\varepsilon 2^{-n}$ holds. Indeed, let $Q$ be a measurable set in $[0, \tau] \times X$ with $(\lambda \otimes \mu)(Q)>\tau-r$ and let

$$
E:=\left\{x \in X: \lambda\left(Q^{x}\right)>\tau-\sqrt{r}\right\}, \quad r \in(0,1) .
$$

Fubini's theorem yields

$$
\tau \mu(E)+(\tau-\sqrt{r})(1-\mu(E))>\tau-r,
$$

whence the inequality $\mu(E)>1-\sqrt{r}$ follows. Let us set

$$
X_{\varepsilon}:=\bigcap_{n=1}^{\infty}\left(E_{n} \cap C_{n}\right) .
$$

We obtain a compact set of measure at least $1-\varepsilon$. Let $x \in X_{\varepsilon}$. We show that the measure $\nu_{t, x}$ with $x \in X_{\varepsilon}$ and $t \geq \varepsilon$ are uniformly tight. Let us fix $r>0$. We take $n$ such that $2^{-n}<r$. Since $X_{\varepsilon} \subset C_{n}$, we have $\nu_{t, x}\left(K_{n}\right)>1-r$ for all $x \in X_{\varepsilon}$ and all $t \geq m_{n}$. Let us consider points $t$ in $[\varepsilon, \tau]$, where $\tau=m_{n}$. Set $K=F\left(Q_{n}\right)$. For all $t \in[\varepsilon, \tau]$ and $x \in X_{\varepsilon}$, by the inclusion $x \in E_{n}$ we have the inequality

$$
\lambda\left(s \in[0,1]: t s \in Q_{n}^{x}\right)=\lambda\left(s \in[0,1]: s \in Q_{n}^{x} / t\right) \geq 1-\varepsilon 2^{-n} / \varepsilon \geq 1-r,
$$

since if a set $A \subset[0, \tau]$ has Lebesgue measure less than $\alpha$, then the intersection of $A / t$ with $[0,1]$ has measure less than $\alpha / t$. Finally, we obtain the following estimate for all $t \in[\varepsilon, \tau]$ and $x \in X_{\varepsilon}$ :

$$
\nu_{t, x}(K)=\nu\left(s \in[0,1]: T_{t s}(x) \in K\right) \geq \nu\left(s \in[0,1]: t s \in Q_{n}^{x}\right) \geq 1-r .
$$

As a common compact set $K_{r}$ for all measures $\nu_{t, x}$ with $t \in[\varepsilon, \infty)$ and $x \in X_{\varepsilon}$ we take the set $K_{n} \cup K$.

We recall (see [7], Ch. 1) that a continuous nonnegative convex function $M$ on $[0,+\infty)$ is called an $\mathrm{N}$-function if the equalities

$$
\lim _{t \rightarrow 0} M(t) / t=0 \quad \text { and } \quad \lim _{t \rightarrow+\infty} M(t) / t=+\infty
$$

hold. Its derivative $M^{\prime}$ increases and has a right inverse function $v$ (if $M^{\prime}$ is continuous and strictly increases, then $v$ is the usual inverse function). The function $M^{*}(t)=\int_{0}^{t} v(s) d s$, which is an N-function as well, is called the adjoint $\mathrm{N}$-function for $M$. Young's inequality $u v \leq M(u)+M^{*}(v)$ holds. For a positive measure $\eta$ on a measurable space $(\Omega, \mathcal{B})$, let $L_{M}(\eta)$ denote the Orlicz class of all $\eta$-measurable functions $\varphi$ such that $M \circ|\varphi| \in L^{1}(\eta)$. For $\varphi \in L_{M}(\eta)$, let

$$
\|\varphi\|_{M}:=\sup \left\{\int_{\Omega} \varphi(\omega) \psi(\omega) \eta(d \omega): \quad \int_{\Omega} M^{*}(|\psi(\omega)|) \eta(d \omega) \leq 1\right\} .
$$

For all $\varphi \in L_{M}(\eta)$ we have $\|\varphi\|_{M}<\infty$ (see [7], §9). We shall say that an N-function $M$ satisfies the $\Delta_{2}$-condition if, for some $k>0$ and $t_{0}>0$, we have $M(2 t) \leq k M(t)$ for all $t \geq t_{0}$. A sufficient condition for this is the concavity of the function $M^{\prime}$ on some ray. If $M$ satisfies the $\Delta_{2}$-condition, then $L_{M}(\eta)$ is a linear space. It is known (see [7], §8) that, for every $\mu$-integrable function $f$, one can find an $\mathrm{N}$-function $M$ satisfying the $\Delta_{2}$-condition such that $M \circ|f| \in L^{1}(\mu)$, i.e., $f \in L_{M}(\mu)$.

Theorem 3. Let $f$ be an $\mathcal{A}$-measurable $\mu$-integrable function and let $M$ be an $N$ function satisfying the $\Delta_{2}$-condition such that $f \in L_{M}(\mu)$. If the density $\varrho$ of the measure $\nu$ has support in the interval $[a, b]$ and $\varrho \in L_{M^{*}}(\lambda)$, where $\lambda$ is Lebesgue measure on $[a, b]$, then, for $\mu$-almost all $x \in X$, we have

$$
\lim _{t \rightarrow+\infty} \int_{0}^{+\infty} f\left(T_{t s}(x)\right) \varrho(s) d s=\mathbb{E}^{\mathcal{T}} f(x),
$$


where $\mathbb{E}^{\mathcal{T}}$ is the conditional expectation with respect to the $\sigma$-algebra $\mathcal{T}$ introduced above. In particular, if the semiflow $\left\{T_{t}\right\}$ is ergodic, then equality (1) holds.

Proof. We may assume that $f \geq 0$. Let $f_{N}=\min (f, N)$ and $g_{N}=f-f_{N}$. For the bounded functions $f_{N}$ our assertion is true, hence, for $\mu$-almost all $x$ and all $N$, we have (2) for $f_{N}$ in place of $f$. Let $h_{t, x, N}(s)=g_{N}\left(T_{t s}(x)\right)$. Let us apply the inequality

$$
\int_{a}^{b} g_{N}\left(T_{t s}(x)\right) \varrho(s) d s \leq\left\|h_{t, x, N}\right\|_{M}\|\varrho\|_{M^{*}}
$$

(see [7], §9). By the $\mu$-integrability of the function $y \mapsto M\left(g_{N}(y)\right)$, we have

$$
\lim _{t \rightarrow \infty} \int_{a}^{b} M\left(g_{N}\left(T_{t s}(x)\right)\right) d s=(b-a) \mathbb{E}^{\mathcal{T}}\left(M \circ g_{N}\right)(x)
$$

for $\mu$-almost all $x$. Let $\varepsilon>0$ be fixed. Since $M$ satisfies the $\Delta_{2}$-condition, according to Theorem 9.4 in [7], there exists $\delta>0$ such that $\|\varphi\|_{M} \leq \varepsilon$ if $\varphi \in L_{M}(\lambda)$ and the integral of $M(\varphi(s))$ over $[a, b]$ does not exceed $\delta$. Since $\lim _{N \rightarrow \infty} \mathbb{E}^{\mathcal{T}} g_{N}(x)=0$ and $\lim _{N \rightarrow \infty} \mathbb{E}^{\mathcal{T}}\left(M \circ g_{N}\right)(x)=0$ for $\mu$-almost all $x$, there exist $N$ and a set $E \in \mathcal{A}$ with $\mu(E)>1-\varepsilon$ such that

$$
(b-a) \mathbb{E}^{\mathcal{T}}\left(M \circ g_{N}\right)(x)<\delta, \quad \mathbb{E}^{\mathcal{T}} g_{N}(x)<\varepsilon, \quad x \in E .
$$

According to what has been said above, for $\mu$-almost all $x \in E$, there exists $T(x, \varepsilon)$ such that for all $t \geq T(x, \varepsilon)$, we have the inequality

$$
\left|\int_{a}^{b} f_{N}\left(T_{t s}(x)\right) \varrho(s) d s-\mathbb{E}^{\mathcal{T}} f_{N}(x)\right|<\varepsilon, \quad \int_{a}^{b} M\left(g_{N}\left(T_{t s}(x)\right)\right) d s<\delta .
$$

The latter estimate yields the inequality $\left\|h_{t, x, N}\right\|_{M} \leq \varepsilon$. According to (3) we obtain

$$
\int_{a}^{b} g_{N}\left(T_{t s}(x)\right) \varrho(s) d s \leq \varepsilon\|\varrho\|_{M^{*}}
$$

Thus, for $\mu$-almost all $x \in E$, if $t \geq T(x, \varepsilon)$, then we have

$$
\left|\int_{a}^{b} f\left(T_{t s}(x)\right) \varrho(s) d s-\mathbb{E}^{\mathcal{T}} f(x)\right|<2 \varepsilon+\varepsilon\|\varrho\|_{M^{*}}
$$

which proves our assertion.

Corollary 1. Suppose that the density $\varrho$ has bounded support in an interval $[a, b]$. Let a function $f \in L^{p}(\mu)$ be $\mathcal{A}$-measurable and let $\varrho \in L^{q}[a, b]$, where $p \in[1,+\infty]$, $q=p /(p-1)$ and $q=\infty$ if $p=1$. Then, for $\mu$-almost all $x \in X$, equality (2) holds. In particular, for any bounded density $\varrho$ with bounded support and every $\mathcal{A}$ measurable $\mu$-integrable function $f$, this equality is true $\mu$-almost everywhere. In the ergodic case, this gives (1).

The assumption of the boundedness of the support of $\varrho$ can be removed if we require a sufficient decay at infinity.

Proposition 1. Suppose that the density $\varrho$ of the measure $\nu$ satisfies the following condition: there exist positive numbers a $(n)$ such that $\varrho(t) \leq a(n)$ if $t \in[n, n+1)$, $n=0,1, \ldots$, and $\sum_{n=1}^{\infty} n a(n)<\infty$. Let $f$ be an $\mathcal{A}$-measurable $\mu$-integrable function. Then, for $\mu$-almost all $x \in X$, equality (2) holds; if the semiflow $\left\{T_{t}\right\}$ is ergodic, then (1) holds. 
Proof. Let $f \geq 0$ and $\varepsilon \in(0,1)$. As above, we write $f$ in the form $f=f_{N}+g_{N}$. There exist $N \in \mathbb{N}$ and a set $A \in \mathcal{A}$ with $\mu(A)>1-\varepsilon$ such that, for every $x \in A$, the relationships

$$
\begin{gathered}
\lim _{t \rightarrow+\infty} \int_{0}^{+\infty} f_{N}\left(T_{t s}(x)\right) \varrho(s) d s=\mathbb{E}^{\mathcal{T}} f_{N}(x)>\mathbb{E}^{\mathcal{T}} f(x)-\varepsilon \\
\lim _{t \rightarrow+\infty} \int_{1}^{2} g_{N}\left(T_{t s}(x)\right) d s=\lim _{t \rightarrow+\infty} \int_{0}^{1} g_{N}\left(T_{t s}(x)\right) d s=\mathbb{E}^{\mathcal{T}} g_{N}(x)<\varepsilon
\end{gathered}
$$

hold. Keeping $x \in A$ fixed, we choose $\tau>1$ such that, for all $t \geq \tau$, the convergent quantities in the above expressions differ from the corresponding limits in at most $\varepsilon$. Notice that

$$
\begin{aligned}
& \int_{n}^{n+1} g_{N}\left(T_{t s}(x)\right) \varrho(s) d s \leq a(n) t^{-1} \int_{t n}^{t(n+1)} g_{N}\left(T_{u}(x)\right) d u \\
& \leq a(n) t^{-1} \int_{t n}^{2 t n} g_{N}\left(T_{u}(x)\right) d u=a(n) n \int_{1}^{2} g_{N}\left(T_{t n s}(x)\right) d s \leq 2 \varepsilon a(n) n
\end{aligned}
$$

whenever $t \geq \tau$. Therefore,

$$
\int_{0}^{+\infty} g_{N}\left(T_{t s}(x)\right) \varrho(s) d s \leq 2 \varepsilon a(0)+2 \varepsilon \sum_{n=1}^{\infty} n a(n),
$$

which yields our assertion.

For periodic systems, the condition on $\varrho$ can be further weakened by requiring only convergence of the series $\sum_{n=1}^{\infty} a(n)$. It is unclear whether the boundedness of $\varrho$ alone is sufficient. It is clear from our reasoning that in order to have (1) in the ergodic case, it suffices to have an estimate of the form

$$
\limsup _{t \rightarrow \infty} \int_{X}|f(z)| \nu_{t, x}(d z) \leq \int_{X}|f(z)| \mu(d z)
$$

for $\mu$-almost all $x$.

Remark 3. (i) In the case where the system is not ergodic, we can pass to the decomposition into ergodic components. If $(X, \mathcal{A}, \mu)$ is a Lebesgue-Rohlin space (see [6], §9.4), then, according to [8], there is a measurable partition of the space $X$ into elements $C$ equipped with conditional probability measures $\mu_{C}$ such that, for almost every (with respect to the measure on the factor-space) partition element $C$, the semigroup $\left\{T_{t}\right\}$ acts in $C$ and is ergodic with respect to the measure $\mu_{C}$. Then, for almost every partition element $C$ and $\mu_{C}$-almost every $x \in C$, Theorem 1 and Theorem 2 hold for the measures $\nu_{t, x}$ provided that the measure $\mu$ in their statements is replaced by $\mu_{C}$. If $X$ is a Souslin space, then the elements $C$ can be chosen Souslin as well.

(ii) The result of V.V. Kozlov and D.V. Treshchev, as well as our Theorem 3, remain valid with the same proofs in more general situations where the individual ergodic theorem holds: see, for example, Theorem 5 in $\S 7$ of Chapter VIII in book [9], where one deals with semigroups on $L^{1}(\mu)$.

This work was supported by the RFBR projects 04-01-00748 and 05-01-02941, the DFG Grant 436 RUS 113/343/0(R), the INTAS Grant 03-51-5018, Forschergruppe spektrale Analysis und stochastische Dynamik and the SFB 701 at the University of Bielefeld. 


\section{REFERENCES}

[1] Kozlov V.V., Treshchev D.V. On new forms of the ergodic theorem. J. Dynam. Control Syst. 2003. V. 9, N 3. P. 449-453.

[2] Kozlov V.V., Treshchev D.V. Evolution of measures in the phase space of nonlinear Hamiltonian systems. Theor. and Math. Phys. 2003. V. 136, N 3. P. $1325-1335$.

[3] Cornfeld I.P., Fomin S.V., Sina ̌ Ya.G. Ergodic theory. Translated from the Russian. Springer-Verlag, New York, 1982

[4] Lukacs E. Characteristic functions. 2nd ed. Hafner Publ., New York, 1970.

[5] Khinchin A.Ya. Continued fractions. Translated from the Russian. Dover, Mineola, 1997.

[6] Bogachev V.I. Foundations of measure theory. V. 1,2. Regular and Chaotic Dynamics, Moscow - Izhevsk, 2003.

[7] Krasnosel'skiı̌ M.A., Rutickiı̌ Ja.B. Convex functions and Orlicz spaces. Translated from the first Russian ed. Noordhoff, Groningen, 1961.

[8] Rohlin V.A. On decomposition of a dynamic system into transitive components. Matem. Sbornik. 1949. V. 25, N 2. P. 235-249 (in Russian).

[9] Dunford N., Schwartz J.T. Linear operators, I. General Theory. Interscience, New York, 1958.

Department of Mechanics and Mathematics, Moscow State University, 119992 Moscow, Russia 